\title{
The improvement of in vivo model (Balb/c mice) for cervical carcinogenesis using diethylstilbestrol (DES)
}

\begin{abstract}
Cervical cancer is the most common gynecological cancer and one of the major causes of female cancer-related death worldwide particularly in developing countries. Thus far, there are a few in vivo models have been developed in investigating this type of cancer. In this study, we induced cervical cancer in Balb/c mice by exploiting the carcinogenic property of diestylstilbestrol (DES). The Balb/c pregnant mice were given subcutaneous (SC) injection of $67 \mu \mathrm{g} / \mathrm{kg}$ body weight of DES on GD 13, and the mice gave birth approximately at gestation day 19-22. Female offspring were reared and the body weight was recorded once weekly. The female offspring were sacrificed at age of 5 months. Upon termination, blood was collected in a plain tube via cardiac puncture and the reproductive tracts were collected and weighed. The reproductive tract sections were stained using H\&E for observation of pathological changes. The progression of disease state was monitored by measuring the level of serum interleukin (IL-6) using the Mouse IL-6 ELISA Assay Kit (BD OptEIA ${ }^{\mathrm{TM}}$, USA). All parameters were compared with Not-induced group. The outcome of this study demonstrated a significant difference in body weight gain, reproductive organ weight, diameter of cervix and the level of serum IL- 6 in the Induced group as compared to the Not-induced group $(\mathrm{P}<$ 0.05). Histopathological findings revealed the presence of adenosis only in the Induced group. It shows that DES could be employed as an agent to induce cervical carcinogenesis in animal model. In addition to that, new potential anti-cancer agents from various sources could be further evaluated using this technique.
\end{abstract}

Keyword: Adenosis; Balb/c mice; Carcinogenesis; Diethylstilbestrol; In vivo model; Interleukin-6 